Discrete Comput Geom 32:521-532 (2004)

DOI: $10.1007 / \mathrm{s} 00454-004-1136-0$

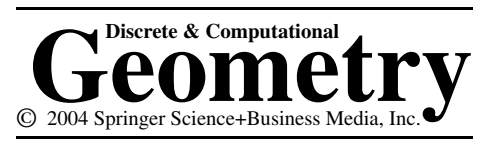

\title{
Mixed Fibre Polytopes
}

\author{
Peter McMullen \\ University College London, Gower Street, \\ London WC1E 6BT, England \\ p.mcmullen@ucl.ac.uk
}

\begin{abstract}
With a slight modification of the original definition by Billera and Sturmfels, it is shown that the theory of fibre polytopes extends to one of mixed fibre polytopes. Indeed, there is a natural surjective homomorphism from the space of tensor weights on polytopes in a euclidean space $\mathbb{V}$ to the corresponding space of such weights on fibre polytopes in a subspace of $\mathbb{V}$. Moreover, these homomorphisms compose in the correct way; this is in contrast to the original situation of the fibre polytope construction.
\end{abstract}

\section{Introduction}

In [2] Billera and Sturmfels introduced the concept of fibre polytopes, which generalized the earlier notion of secondary polytopes of [4] due to Gel'fand et al. (see also [1] and, for general background as well, [21]). In [8] McDonald investigated how to express the solutions of $k$ polynomial equations in terms of fractional power series for $k$ of the variables in terms of the remainder, and observed that these solutions were associated with a certain fibre polytope of the Newton polytope of the system. He also asked whether, in fact, there exist corresponding mixed fibre polytopes (of the Newton polytopes of the individual polynomials) with which the solutions could be better associated. A partial answer to this question had already been given in [17].

In this paper we address the general theoretical question, rather than the specific problem raised in [8]. We slightly change the original definition of fibre polytope, and show that it leads naturally to mixed fibre polytopes. This is so because the new construction is a weakly continuous translation covariant valuation, and so admits polynomial expansions of non-negative (Minkowski) linear combinations of polytopes.

In fact, the theory is richer than this. It turns out that there are surjective homomorphisms between the appropriate spaces of tensor weights on polytopes (the background for these can be found in [14] and [16]). The construction is direct; a consequence is that the coefficients of the polynomials in the first weight space of the image correspond to genuine polytopes-these are the mixed fibre polytopes initially asked for. 
The resulting homomorphisms between tensor weight spaces compose in the right way. It is important to note that the original fibre polytope construction is not categorical, even when the definition is phrased in the more general context introduced in [15]. The results of this paper make it clear why this is so.

Finally, applications are given to the mixed polytopes of Schneider [19] and to the fibre polytope of a zonotope; the latter is identified as the projection body of the associated zonotope.

For general information about convexity, and convex polytopes in particular, we refer the reader to [6], [7] and [21].

\section{Valuations and Weak Continuity}

Our first task is to redefine the fibre polytope, and then establish basic properties of the construction. The setting in this section is the following. For simplicity, we work in euclidean spaces. In fact, everything carries over to inner product spaces over a field $\mathbb{F}$ which is square-root-closed; for many reasons (which we do not go into here), a good choice of such a field is that of the real algebraic numbers. (Yet further generalization is possible, but this introduces complications which we prefer to avoid.)

So far as is possible, we wish to stay close to what we did in [15]. However, the context of this paper, particularly that of tensor weights, almost demands that we work in euclidean spaces. So, let $\mathbb{V}$ be a euclidean space, and let $\mathbb{X} \leq \mathbb{V}$ be a proper subspace; we write $\Phi: \mathbb{X} \rightarrow \mathbb{V}$ for the injection, so that $\Phi$ implicitly refers to $\mathbb{X}$ and $\mathbb{V}$ as well. Indeed, it is appropriate to think of $\Phi$ as a linear isometry, embedding $\mathbb{X}$ in $\mathbb{V}$.

Let $\mathbb{Y}:=\mathbb{X}^{\perp}$ be the orthogonal complement of $\mathbb{X}$ in $\mathbb{V}$. If we write $\Psi$ for the orthogonal projection on $\mathbb{Y}$, then we have a short exact sequence

$$
\mathbb{O} \longrightarrow \mathbb{X} \stackrel{\Phi}{\longrightarrow} \mathbb{V} \stackrel{\Psi}{\longrightarrow} \mathbb{Y} \longrightarrow \mathbb{O}
$$

of spaces and linear mappings; recall that this means that $\Phi$ is injective, $\Psi$ is surjective and $\operatorname{im} \Phi=\operatorname{ker} \Psi$. Our assumption is that neither $\Phi$ nor $\Psi$ is trivial or an isomorphism.

The sequence of dual spaces and mappings

$$
\mathbb{0} \longleftarrow \mathbb{X}^{*} \stackrel{\Phi^{*}}{\longleftarrow} \mathbb{V}^{*} \stackrel{\Psi^{*}}{\longleftarrow} \mathbb{Y}^{*} \longleftarrow \mathbb{O}
$$

is also short exact. In our context we invariably identify dual spaces, so that $\mathbb{X}^{*}=\mathbb{X}$, and so on. This implies that $\Phi^{*}$ is an orthogonal projection on $\mathbb{X}$, and that $\Psi^{*}$ is a linear isometry.

The dimension which is most important later is that of $\mathbb{Y}$; we write $m:=\operatorname{dim} \mathbb{Y}$.

Let $\mathcal{P}(\mathbb{V})$ denote the family of (non-empty convex) polytopes in $\mathbb{V}$, and let $P \in \mathcal{P}(\mathbb{V})$. Then, as we redefine the term, the fibre polytope of $P$ with respect to $\Phi$ is

$$
\operatorname{fib}(P ; \Phi):=\int_{\mathbb{Y}}((P-y) \cap \mathbb{X}) d y,
$$

where the integral is with respect to the usual Lebesgue measure on $\mathbb{Y}$; conventionally, an empty integrand is replaced by $\{o\}$, with $o$ the zero vector. Observe that, when it is non-empty, $(P-y) \cap \mathbb{X}$ is the fibre of $P$ over $y \in P \Psi$ under the projection $\Psi$. 
We may think of this integral in two ways. First, we can define it, as usual, as a limit of Riemann sums, with polytopes (fibres) standing in for functions, so that the Riemann sums are Minkowski linear combinations (see below). Alternatively, if $h(P, u)$ denotes the support functional of $P$ in direction $u$, then

$$
h(\operatorname{fib}(P ; \Phi), u)=\int_{P \Psi} h(((P-y) \cap \mathbb{X}), u) d y
$$

for each $u \in \mathbb{X}$ (or, perhaps better, in $\mathbb{X}^{*}$, but we do not insist on this distinction). The notation is intended to be reminiscent of that of [15]; we have modified it to indicate that fib denotes a particular polytope, whereas Fib corresponds to a whole strong isomorphism class. Moreover, we retain the dependence of the notation on the injection $\Phi$ (rather than placing it on $\mathbb{X}$ ), because this facilitates what we wish to do later. (We do not repeat the familiar argument which shows that $\operatorname{fib}(P ; \Phi)$ is a polytope.)

Now, it is obvious from the definition that, for $t \in \mathbb{V}$, we have

$$
\operatorname{fib}(P+t ; \Phi)=\operatorname{fib}(P ; \Phi)+\operatorname{vol}(P \Psi)\left(t \Phi^{*}\right),
$$

recalling that $\Psi$ and $\Phi^{*}$ are the appropriate orthogonal projections. (The component of $t$ in $\mathbb{Y}$ does not make a contribution, because it is cancelled by translating the fibres.) This tells us that the mapping fib $(\cdot ; \Phi)$ is translation covariant, a term which we return to at the end of the section. We also observe that fib $(\cdot ; \Phi)$ is (non-negative) homogeneous of degree $m+1$ (with $m=\operatorname{dim} \mathbb{Y}$ as before), meaning that $\operatorname{fib}(\lambda P ; \Phi)=\lambda^{m+1} \operatorname{fib}(P ; \Phi)$ for each $\lambda \geq 0$, because (2.1) implies that we are integrating a linear (homogeneous of degree 1) function with respect to an $m$-dimensional measure (this accords with (2.2)).

However, to develop a more general theory, we need various other properties of fib. We recall (see, for example, [12]) that a valuation on $\mathcal{P}(\mathbb{V})$ is a mapping $\varphi$, taking values in some abelian (semi)group, such that

$$
\varphi(P \cup Q)+\varphi(P \cap Q)=\varphi(P)+\varphi(Q)
$$

whenever $P, Q \in \mathcal{P}(\mathbb{V})$ are such that $P \cup Q$ is convex (and so also lies in $\mathcal{P}(\mathbb{V})$ ). The core result on which the paper depends is

Theorem 2.1. The mapping fib $(\cdot ; \Phi): \mathcal{P}(\mathbb{V}) \rightarrow \mathcal{P}(\mathbb{X})$ is a valuation.

Proof. Suppose that $P, Q \in \mathcal{P}(\mathbb{V})$ are such that $P \cup Q$ is convex. First, for each $y \in \mathbb{Y}$, we see that

$$
((P-y) \cap \mathbb{X}) \cup((Q-y) \cap \mathbb{X})=((P \cup Q)-y) \cap \mathbb{X}
$$

is convex. Next,

$$
h(P \cup Q, u)=\max \{h(P, u), h(Q, u)\}, \quad h(P \cap Q, u)=\min \{h(P, u), h(Q, u)\},
$$

so that

$$
h(P \cup Q, u)+h(P \cap Q, u)=h(P, u)+h(Q, u) .
$$

The same therefore holds for the fibres over each $y \in \mathbb{Y}$, now taking $u \in \mathbb{X}$, with the appropriate interpretation whenever one or other fibre is empty. Last, integrating yields the claimed result. 
We can think of a polytope either as the convex hull of finitely many points, or as a bounded intersection of finitely many closed half-spaces. A function $\varphi$ (usually, in this context, a valuation) on $\mathcal{P}(\mathbb{V})$ is weakly continuous if it is continuous with respect to parallel displacement of these bounding half-spaces. We then have

Theorem 2.2. The mapping fib $(\cdot ; \Phi): \mathcal{P}(\mathbb{V}) \rightarrow \mathcal{P}(\mathbb{X})$ is weakly continuous.

Proof. This is clear: in fact, the support functional $h(\cdot, u)$ is not just weakly continuous-it is continuous with respect to the Hausdorff metric topology on polytopes.

We can now appeal to general theory (see [10] and [16]—a preliminary account of part of the latter appeared in [14]). Let $\varphi$ be a valuation on $\mathcal{P}(\mathbb{V})$. We call $\varphi$ translation invariant if $\varphi(P+t)=\varphi(P)$ for each $t \in \mathbb{V}$ and $P \in \mathcal{P}(\mathbb{V})$. Following [18], we say that $\varphi$ is polynomial of degree $r(\geq 1)$ if $\psi(\cdot, t)$, given by $\psi(P, t):=\varphi(P+t)-\varphi(P)$, is polynomial of degree $r-1$ for each $t \in \mathbb{V}$; the definition starts with the case $r=0$ of translation invariance. A valuation which is polynomial of some degree is called translation covariant. We denote by $\mathcal{V}(\mathbb{V})$ the family of weakly continuous translation covariant valuations on $\mathcal{P}(\mathbb{V})$ (strictly speaking, we should indicate the target space, but this can always be understood from the context).

A core result from [16] is the following (see also [18] for closely related ideas): if $\varphi \in \mathcal{V}(\mathbb{V})$, then $\varphi$ admits polynomial expansions in (Minkowski) linear combinations of polytopes. This means that if $P_{1}, \ldots, P_{k} \in \mathcal{P}(\mathbb{V})$ and $\lambda_{1}, \ldots, \lambda_{k} \geq 0$, then $\varphi\left(\lambda_{1} P_{1}+\cdots+\right.$ $\lambda_{k} P_{k}$ ) is a polynomial in $\lambda_{1}, \ldots, \lambda_{k}$. In general, if $\varphi$ is a polynomial valuation of degree $r$, then there exist such polynomial expansions of degree $\operatorname{dim} \mathbb{V}+r$ with rational scalars $\lambda_{j}$; compare Section 3.5 of [12], where the crucial result should have read $Z^{d+r} \leq T^{r}$ (the direction of the inequality was misstated). Weak continuity then extends the expansions to real scalars. In particular, this result holds for fib $(\cdot ; \Phi) \in \mathcal{V}(\mathbb{V})$, which already goes most of the way towards establishing what was announced in Section 1.

\section{Tensor Weights}

The general separation theory of [16] shows that the appropriate context for a discussion of weakly continuous translation covariant valuations is that of tensor weights. We merely sketch the background here, referring the reader to [10], [14] and [16] for further details. We should remark at the outset that what we want to do subsequently strongly suggests that we work in euclidean spaces, as we indicated in Section 1.

If $P \in \mathcal{P}(\mathbb{V})$, then $\mathcal{F}(P)$ denotes the family of all non-empty faces of $P$ (including $P$ itself). If $F \in \mathcal{F}(P)$, then we also write $F \leq P$. Further, $F_{\|}:=\operatorname{lin}(F-F)$ is the linear subspace parallel to $F$. We propose to write $F \triangleleft G$ to mean that $F$ is a facet of $G$, so that $F<G$ with $\operatorname{dim} G-\operatorname{dim} F=1$; we then denote by $u(F, G)$ the unit outer normal vector to $G$ at $F$ lying in $G_{\|}$.

Let $\mathbb{T}=\mathbb{T}(\mathbb{V})$ be the algebra of symmetric tensors over $\mathbb{V}$. For simplicity of notation, if $a, b \in \mathbb{T}$, then we write $a b:=a \otimes_{\text {sym }} b$, where $\otimes_{\text {sym }}$ denotes the symmetric tensor product over $\mathbb{R}$. (By contrast, when we write $\otimes$ unadorned, then we mean the usual tensor product over the integers $\mathbb{Z}$.) 
Let $\langle\cdot, \cdot\rangle$ be the inner product in $\mathbb{V}$. Then we say that $a: \mathcal{F}(P) \rightarrow \mathbb{T}$ is a (tensor) weight if the Green-Minkowski connexions (GMC) hold, namely,

$$
\sum_{F \triangleleft G} a(F)\langle u(F, G), t\rangle=a(G) t
$$

for each $G \leq P$ and each $t \in G_{\|}$. The idea is to generalize the relations which prevail for the $r$-class $p_{r}$ of a polytope $P$, which is defined as follows. The $s$-tensorial $M_{s}(P)$ of a polytope $P$ is given by

$$
M_{s}(P):=\frac{1}{s !} \int_{P} x^{s} d x,
$$

where the integration is with respect to the usual Lebesgue measure in aff $P$; by convention, we write $M_{s}(P):=0$ if $s<0$. Then

$$
p_{r}(F):=M_{r-\operatorname{dim} F}(F)
$$

for each $F \leq P$ and $r \geq 0$; note that $p_{r}(\lambda F)=\lambda^{r} p_{r}(F)$ for each $\lambda \geq 0$, with $0^{0}:=1$, so that $p_{r}$ is homogeneous of degree $r$. Observe that if (3.1) holds, then we can recover $a(G)$ using a single (non-zero) $t \in G_{\|}$, because we can embed $\mathbb{T}$ in its field of fractions.

We should point out that the GMC for $p_{r}$ arises from Green's theorem (see [5]), in the form

$$
\sum_{F \triangleleft G} M_{s}(F)\langle u(F, G), t\rangle=M_{s-1}(G) t .
$$

It generalizes Minkowski's theorem on facet-areas of polytopes, which accounts for the nomenclature.

It is clear that the set $\mathcal{W}(P)$ of tensor weights on $P$ has the structure of a vector space. In fact, it is actually an algebra. We do not need the multiplicative structure here (indeed, that originally defined in [14] turns out to be inappropriate here), but one geometric implication of the GMC is that tensor weights behave correctly under linear mappings (this will appear in a different guise in Section 4). There is then induced a multiplication $\mathcal{W}(P) \otimes \mathcal{W}(Q) \rightarrow \mathcal{W}(P+Q)$; the identity $i=i_{Q} \in \mathcal{W}(Q)$ is the 0 -weight which takes the value $i(x)=1$ for each vertex $x$ of the polytope $Q$, and then multiplication by $i$ embeds $\mathcal{W}(P)$ into $\mathcal{W}(P+Q)$. This enables us to regard weights on several polytopes as weights on just one which, when convenient, we can even take to be simple. Speaking more technically, we can proceed to the direct limit, to obtain the tensor weight algebra $\mathcal{W}(\mathbb{V})$.

There is a more abstract formulation of weights. For each subspace $\mathbb{L} \leq \mathbb{V}$, let $\widehat{\Sigma}_{\mathbb{L}}$ be the abelian group with a generator $\langle K\rangle=\langle K\rangle_{\mathbb{L}}$, called its class, for each polyhedral cone $K$ (with apex $o$ ) in $\mathbb{L}$, satisfying the relations

$$
\left\langle H^{-} \cap K\right\rangle+\left\langle H^{+} \cap K\right\rangle=\langle K\rangle
$$

whenever $H$ is a hyperplane in $\mathbb{L}$ bounding the two (closed) half-spaces $H^{-}$and $H^{+}$; thus, if $K$ is a cone in $\mathbb{L}$ with $\operatorname{dim} K<\operatorname{dim} \mathbb{L}$, then $\langle K\rangle_{\mathbb{L}}=0$. The (simple) cone group in $\mathbb{V}$ is then the direct sum

$$
\widehat{\Sigma}(\mathbb{V}):=\bigoplus_{\mathbb{L} \leq \mathbb{V}} \widehat{\Sigma}_{\mathbb{L}}
$$


If $N(F, P)$ is the normal cone to a polytope $P$ at its face $F$, then we write $\widehat{n}(F, P):=$ $\langle N(F, P)\rangle$ for its class, always (in this context) taken relative to the linear hull lin $N(F, P)$. An important result from [16] is then

Proposition 3.1. The image of the mapping from $\mathcal{W}(\mathbb{V})$ to $\mathbb{T} \otimes \widehat{\Sigma}(\mathbb{V})$, induced by

$$
a \mapsto \sum_{F \leq P} a(F) \otimes \widehat{n}(F, P)
$$

for each $P \in \mathcal{P}(\mathbb{V})$, is universal for $\mathcal{V}(\mathbb{V})$.

What this means is that the mapping of the proposition is an injective homomorphism, and that every weakly continuous translation covariant valuation induces a corresponding homomorphism on its image in $\mathbb{T} \otimes \widehat{\Sigma}(\mathbb{V})$.

To end this section we note another important fact from [16].

Proposition 3.2. The weight algebra $\mathcal{W}(\mathbb{V})$ is generated by the (1-) classes of (simple) polytopes in $\mathcal{P}(\mathbb{V})$.

\section{The Fibre Map}

We have already observed that we have polynomial expansions in $\mathcal{P}(\mathbb{X})$ of fibre polytopes of Minkowski linear combinations of polytopes in $\mathcal{P}(\mathbb{V})$. Indeed, appealing to the results of Section 3, and Theorems 2.1 and 2.2, we obtain analogous results for tensor weights. In this section we show how a weight on $P$ induces one on $\operatorname{fib}(P ; \Phi)$.

Let $P \in \mathcal{P}(\mathbb{V})$, and let $a \in \mathcal{W}(P)$. Using Proposition 3.2, we may assume that $P$ is simple, and that $a$ is a tensorial. A face $F \leq P$ will make a contribution only if $F \Psi$ is full-dimensional in $\mathbb{Y}$, so that $\operatorname{dim}(F \Psi)=m$. (Such a face we call general; the rest are singular.) We wish to apply Fubini's theorem, but we need a scaling factor $\kappa(F, \Psi)>0$, called the volume ratio, defined as follows. Pick an orthonormal basis $\left\{b_{1}, \ldots, b_{k}\right\}$, say, of $F_{\|}$, so that $\left\{b_{m+1}, \ldots, b_{k}\right\}$ is a basis of $F_{\|} \cap \mathbb{X}$. Let $C$ be the unit $m$-cube based on $\left\{b_{1}, \ldots, b_{m}\right\}$; then $\kappa(F, \Psi)$ is the volume of $C \Psi$. (We have slightly varied our usual definition here. With the convention of [13] and [16], we should really write $\kappa\left(F \cap(F \cap \mathbb{X})^{\perp}, \Psi\right)$ instead here; compare the definition of $\kappa\left(K, \Phi^{*}\right)$ below.)

We deduce that

$$
\int_{F \Psi} M_{s}(F \cap(\mathbb{X}+y)) d y=\kappa(F, \Psi) M_{s}(F) .
$$

Now

$$
M_{s}((F-y) \cap \mathbb{X})=\left(M_{s}(F \cap(\mathbb{X}+y))\right) \Phi^{*},
$$

and so, taking the orthogonal projection $\Phi^{*}$ outside the integral, we see that $F$ contributes $\kappa(F, \Psi) M_{s}(F) \Phi^{*}$ to the corresponding weight on $\operatorname{fib}(P ; \Phi)$. Notice, by the way, that GMC holds for each such fibre, and so the corresponding weight contribution to fib $(P ; \Phi)$ must also satisfy GMC; that is, it is indeed a weight. 
It is clear that the same calculation carries over to a singular face $F$; here we have $\kappa(F, \Psi)=0$.

Now the normal fan of $\operatorname{fib}(P ; \Phi)$, which consists of the complex of normal cones to its faces, is just the common refinement of the fan induced by the projection of the normal fan of $P$ under $\Phi^{*}$ (see, for example, [15]). If $F \leq P$, then the contribution of its normal cone is thus $N(F, P) \Phi^{*}$. We observe that the scaling factor $\kappa(F, \Psi)$ gives the corresponding volume ratio of the projection here as well; compare here [11], although the situations are not exactly the same. Thus, if $K$ is a $k$-dimensional cone in $\mathbb{V}$, then the scaling factor $\kappa\left(K, \Phi^{*}\right)$ is the volume ratio of the projection $\Phi^{*}$ on $K$, namely,

$$
\kappa\left(K, \Phi^{*}\right):=\operatorname{vol}_{k}\left(C \Phi^{*}\right) / \operatorname{vol}_{k} C,
$$

where $C$ is a $k$-dimensional box in $K_{\|}$. We extend $\Phi^{*}$ to $\widehat{\Sigma}(\mathbb{V})$ in the natural way by $\langle K\rangle \Phi^{*}:=\left\langle K \Phi^{*}\right\rangle$; we should interpret the latter here as 0 if $\Phi^{*}$ is singular on $K$, but this is also taken care of by the volume ratio. (Observe that the latter is now the conventional definition.) Our conclusion is the following:

Theorem 4.1. Define $\bar{\Phi}: \mathbb{T}(\mathbb{V}) \otimes \widehat{\Sigma}(\mathbb{V}) \rightarrow \mathbb{T}(\mathbb{X}) \otimes \widehat{\Sigma}(\mathbb{X})$ on its generators by

$$
(a \otimes\langle K\rangle) \bar{\Phi}:=\kappa\left(K, \Phi^{*}\right)\left(a \Phi^{*}\right) \otimes\langle K\rangle \Phi^{*} .
$$

Then $\bar{\Phi}$ induces a vector space homomorphism from $\mathcal{W}(\mathbb{V})$ to $\mathcal{W}(\mathbb{X})$. If a $\in \mathcal{W}(P)$ for some $P \in \mathcal{P}(\mathbb{V})$, then a $\bar{\Phi} \in \mathcal{W}(\mathrm{fib}(P ; \Phi))$.

We call $\bar{\Phi}$ the fibre map. Of course, $\bar{\Phi}$ is certainly not an algebra homomorphism, if only because it does not preserve degrees. An important property of $\bar{\Phi}$ is the following:

Theorem 4.2. $\quad$ The fibre map $\bar{\Phi}: \mathcal{W}(\mathbb{V}) \rightarrow \mathcal{W}(\mathbb{X})$ is surjective.

Proof. It suffices to consider a weight $b$ on a (simple) polytope $Q \in \mathcal{P}(\mathbb{X})$. Let $C \in$ $\mathcal{P}(\mathbb{Y})$ be a unit cube (or, indeed, any polytope of volume 1), and let $c$ be the canonical $d$-weight on $C$. Let $P:=Q \times C \in \mathcal{P}(\mathbb{V})$, and let $a:=b \times c$ be the product weight on $P$ (that is, $a(F \times G)=b(F) c(G)$ for $F \leq Q$ and $G \leq C)$. Then $a \bar{\Phi}=b$. Observe that the components of $c$ on proper faces of $C$ vanish under $\bar{\Phi}$, because they are nonscalar tensors in $\mathbb{T}(\mathbb{Y})$. Thus the only part of $c$ which survives to contribute to $a \bar{\Phi}$ is $b(\cdot) \times c(C)=b$, as required.

Remark 4.3. The fibre map is far from injective, of course. Naturally, weights arising from polytopes $P$ such that $P \Psi$ is not full-dimensional in $\mathbb{Y}$ lie in the kernel, but it is easy to see that these are not the only ones which do.

Remark 4.4. We should note that the definition of $\bar{\Phi}$ is somewhat strange from the viewpoint of the associated mappings. The dual mapping $\Phi^{*}$ certainly induces a mapping on the cone group in the correct direction. However, $\Phi^{*}$ should not be acting on $\mathbb{T}(\mathbb{V})$; rather, we should have $\Phi$ acting on $\mathbb{T}(\mathbb{X})$. 
Remark 4.5. When we pass to the algebra $\Omega(\mathbb{V}):=\mathcal{W}(\mathbb{V}) / \mathcal{T}(\mathbb{V})$ of scalar weights, where $\mathcal{T}(\mathbb{V})$ is the ideal of $\mathcal{W}(\mathbb{V})$ generated by $\mathbb{T} \backslash \mathbb{R}$ (or by $\mathbb{V}$ as the space of 1-tensors), then the problem mentioned in Remark 4.4 disappears. Yet now, under $\bar{\Phi}$, a weight supported on $r$-faces of $P$ turns into one supported by $(r-m)$-faces. However, the codimension of these faces is the same. Now, for a simple $n$-polytope $P$, the spaces $\Omega_{r}(P)$ (those which are homogeneous of degree $r$ ) and $\Omega_{n-r}(P)$ are duals under the multiplication in $\Omega(P)$. When we have dual spaces, then we expect a corresponding duality of maps. In [16] we show that maps such as $\bar{\Phi}$ are the appropriate duals (indeed, in a somewhat more general context).

\section{The First Weight Space}

We now apply the results of Section 4 to give an explicit description of the fibre polytope $\operatorname{fib}(P ; \Phi)$ itself. The canonical 1-weight $q$ of a polytope $Q$ has two components: a vector (1-tensor) $q(v):=v$ on each vertex $v$, and a scalar (0-tensor) edge-length $q(E):=$ $\|v-w\|$ for each edge $E=\operatorname{conv}\{v, w\}$. (The GMC is easy to verify directly here.)

When $Q=\operatorname{fib}(P ; \Phi)$, contributions to its vertices come only from vertices of the fibres, while those to the lengths of its edges arise solely from edges of the fibres. This is in contrast to its faces of dimension at least 2, which may well arise as sums of faces of lower dimensions. We treat the two cases separately.

For vertices, we can ask for that which is the face in a general normal direction $u \in \mathbb{X}$. This lies in the interior of a cone of the normal fan of $\operatorname{fib}(P ; \Phi)$, and so in the interiors of certain cones $N(F, P) \Phi^{*}$, with $F \leq P$ an $m$-face. Writing $m(F):=M_{1}(F)$ for its moment vector, $F$ then contributes $\kappa(F, \Psi) m(F) \Phi^{*}$ to the vertex of fib $(P ; \Phi)$. That is,

Proposition 5.1. If $u \in \mathbb{X}$ is a general direction, then the vertex of $\operatorname{fib}(P ; \Phi)$ in direction $u$ is

$$
\sum_{u \in \operatorname{int}\left(N(F, P) \Phi^{*}\right)} \kappa(F, \Psi) m(F) \Phi^{*} .
$$

The formula for edge-lengths is very similar; we write vol $=M_{0}$ for the appropriate dimensional volume.

Proposition 5.2. If $u \in \mathbb{X}$ is a direction vector lying in the relative interior of a cone of the normal fan of $\operatorname{fib}(P ; \Phi)$ of codimension exactly 1 , then the edge of $\operatorname{fib}(P ; \Phi)$ in direction $u$ has length

$$
\sum_{u \in \operatorname{relint}\left(N(F, P) \Phi^{*}\right)} \kappa(F, \Psi) \operatorname{vol}(F) .
$$

Of course, $\Phi^{*}$ is not needed in this formula.

\section{Mixed Fibre Polytopes}

The results of Section 5 describe the vertices and edge-lengths of a fibre polytope. We now go on to define and describe mixed fibre polytopes. 
The original definition (2.1) and Propositions 5.1 and 5.2 show that $\operatorname{fib}(P ; \Phi)$ is homogeneous of degree $m+1$ in $P$ (with $m:=\operatorname{dim} \mathbb{Y}$ as before). Thus the most general mixed fibre polytope will have $m+1$ arguments (in each of which it is linear), say $P_{0}, \ldots, P_{m}$, and the appropriate definition is: the mixed fibre polytope $\operatorname{fib}\left(P_{0}, \ldots, P_{m} ; \Phi\right)$ is the coefficient of $(m+1) ! \lambda_{0} \cdots \lambda_{m}$ in fib $\left(\lambda_{0} P_{0}+\cdots+\lambda_{m} P_{m} ; \Phi\right)$, where $\lambda_{0}, \ldots, \lambda_{m}$ are non-negative variables. As usual in such a context, the normalization ensures that

$$
\operatorname{fib}(P ; \Phi)=\operatorname{fib}(P, \ldots, P ; \Phi) .
$$

For the vertices, pick a general direction $u \in \mathbb{X}$. This gives faces $F=F_{0}+\cdots+F_{m}$ of $P:=P_{0}+\cdots+P_{m}$ of dimension at most $m$ (recall that the fibre polytopes fib $\left(\lambda_{0} P_{0}+\right.$ $\left.\cdots+\lambda_{m} P_{m} ; \Phi\right)$ are all strongly isomorphic for $\left.\lambda_{0}, \ldots, \lambda_{m}>0\right)$. The corresponding vertex of $\operatorname{fib}\left(P_{0}, \ldots, P_{m} ; \Phi\right)$ is then

$$
\sum_{u \in \operatorname{int}\left(N(F, P) \Phi^{*}\right)} \kappa(F, \Psi) m\left(F_{0}, \ldots, F_{m}\right) \Phi^{*}
$$

with $m\left(F_{0}, \ldots, F_{m}\right)$ the mixed moment, and the sum extending over such faces $F$.

The expression for edge-lengths is, in a sense, easier to visualize. Now, our direction vector $u$ lies in a cone of the normal fan of $\operatorname{fib}(P ; \Phi)$ of codimension exactly 1 . Then the edge of $\operatorname{fib}(P ; \Phi)$ in direction $u$ has length

$$
\sum_{u \in \operatorname{relint}\left(N(F, P) \Phi^{*}\right)} \kappa(F, \Psi) V\left(F_{0}, \ldots, F_{m}\right),
$$

with $V\left(F_{0}, \ldots, F_{m}\right)$ the usual mixed volume.

There is an important consequence of the latter formula.

Theorem 6.1. There exists a mixed fibre polytope fib $\left(P_{0}, \ldots, P_{m} ; \Phi\right)$.

What this means is the following. Regarded as a 1-weight, it is not obvious a priori that $\operatorname{fib}\left(P_{0}, \ldots, P_{m} ; \Phi\right)$ takes non-negative values on edges of fib $(P ; \Phi)$. However, it clearly does, because the mixed volumes $V\left(F_{0}, \ldots, F_{m}\right)$ are non-negative.

\section{Iterated Fibre Polytopes}

In [3] Billera and Sturmfels discussed the iteration of the fibre polytope construction. However, it was observed there that if $\Theta: \mathbb{W} \rightarrow \mathbb{X}$ and $\Phi: \mathbb{X} \rightarrow \mathbb{V}$ are (in our terms) linear isometries, then it is generally the case that

$$
\mathrm{fib}(P ; \Theta \Phi) \neq \operatorname{fib}(\operatorname{fib}(P ; \Phi) ; \Theta) .
$$

Indeed, the former will be (up to a scaling factor) a Minkowski summand of the latter.

What is really happening is explained by Theorems 4.1 and 4.2. On the level of weights, that theorem clearly implies

Theorem 7.1. Let $\Theta: \mathbb{W} \rightarrow \mathbb{X}$ and $\Phi: \mathbb{X} \rightarrow \mathbb{V}$ be linearisometries, and let $\bar{\Theta}: \mathcal{T}(\mathbb{X}) \rightarrow$ $\mathcal{T}(\mathbb{W})$ and $\bar{\Phi}: \mathcal{T}(\mathbb{V}) \rightarrow \mathcal{T}(\mathbb{X})$ be the induced fibre maps on the weight spaces. Then

$$
\overline{\Theta \Phi}=\bar{\Phi} \bar{\Theta} .
$$


The reason that the mapping fib does not behave as well is that a polytope is naturally identifiable with its canonical 1-weight, whereas, as we have seen, the new 1-weights on fib $(P ; \Phi)$ in $\mathbb{X}$ arise from the image of $(m+1)$-weights on $P$ under the fibre map $\bar{\Phi}$.

\section{Mixed Polytopes}

Building on earlier work, in [19] Schneider described what he called mixed polytopes of $P_{1}, \ldots, P_{k}$ in a euclidean space $\mathbb{E}$; their support functionals are the (suitably normalized) coefficients in the polynomial expansion in variables $\lambda_{1}, \ldots, \lambda_{k} \geq 0$ of

$$
\int_{\mathbb{E}^{k-1}} h\left(\left(\lambda_{1} P_{1}+x_{1}\right) \cap \cdots \cap\left(\lambda_{k-1} P_{k-1}+x_{k-1}\right) \cap \lambda_{k} P_{k}, \cdot\right) d x_{1} \cdots d x_{k-1},
$$

with our previous convention that sets $h(\emptyset, \cdot)=0$. In [15] we modified his original definition (which is not quite symmetric in $P_{1}, \ldots, P_{k}$ ) to bring it into line with the general constructions which we introduced there.

Schneider himself related his mixed polytopes to particular cases of fibre polytopes. Our formulation is the following. We have $k$ polytopes $P_{1}, \ldots, P_{k}$ in $d$-dimensional euclidean space $\mathbb{E}$. Then, by (our) definition, the mixed polytopes of $P_{1}, \ldots, P_{k}$ are the (suitably normalized) coefficients in the expansion of fib $\left(\lambda_{1} P_{1} \times \cdots \times \lambda_{k} P_{k} ; \Phi\right)$ as a polynomial in the variables $\lambda_{1}, \ldots, \lambda_{k} \geq 0$, where $\Phi: \mathbb{E} \rightarrow \mathbb{V}:=\mathbb{E}^{k}$, given by

$$
x \Phi:=(x, \ldots, x),
$$

is the diagonal mapping; this is an isometric injection only up to a scaling factor, but will serve the purpose. The orthogonal complement of $\mathbb{X}:=\operatorname{im} \Phi$ in $\mathbb{V}$ is

$$
\mathbb{Y}=\left\{y=\left(x_{1}, \ldots, x_{k}\right) \in \mathbb{V} \mid x_{1}+\cdots+x_{k}=o\right\} .
$$

We obtain a fibre over the typical point $y \in \mathbb{Y}$ just when

$$
\left(\lambda_{1} P_{1}-x_{1}\right) \cap \cdots \cap\left(\lambda_{k} P_{k}-x_{k}\right) \neq \emptyset ;
$$

note that this restores the symmetry among $P_{1}, \ldots, P_{k}$. The dual of $\Phi$ is the sum mapping $\Phi^{*}: \mathbb{V} \rightarrow \mathbb{E}$, given by

$$
\left(x_{1}, \ldots, x_{k}\right) \Phi^{*}:=x_{1}+\cdots+x_{k}
$$

again, up to a scaling factor, this is an orthogonal projection.

With these ingredients, and noting that (in the notation of Section 6) $m=(k-1) d$, we see that, in principle, we can work out straightforwardly the vertices and edge-lengths of the mixed polytopes. We observe (as did Schneider) that the coefficients are indeed genuine polytopes.

\section{Zonotopes}

The fibre polytope of a cube was explicitly calculated in [2]. In [15] we obtained this fibre polytope (up to strong isomorphism) as the projection body of a certain zonotope. 
(Several articles in [7] mention projection bodies, and so we give this as a general reference on the topic.) We claimed there that the result held on a more metrical level; here we expand on this.

In the context of this paper, we are considering isometric injections and orthogonal projections. Rather than just take the unit $n$-cube in the $n$-dimensional space $\mathbb{V}$, we vary its edges, and define the box

$$
C=C\left(\lambda_{1}, \ldots, \lambda_{n}\right):=\sum_{i=1}^{n} \frac{1}{2} \lambda_{i}\left[-e_{i}, e_{i}\right],
$$

with $\left(e_{1}, \ldots, e_{n}\right)$ an ordered orthonormal basis of $\mathbb{V}$ and $\lambda_{1}, \ldots, \lambda_{n}>0$. Thus the edgelengths of the scaled box $C$ are $\lambda_{1}, \ldots, \lambda_{n}$. We calculate the fibre polytope of $C$, using our previous results.

As before, we think of $\mathbb{X}$ and $\mathbb{Y}$ as orthogonal complementary subspaces of $\mathbb{V}$, with $\operatorname{dim} \mathbb{Y}=m$ (since $\operatorname{dim} \mathbb{V}=n$, this gives $\operatorname{dim} \mathbb{X}=n-m)$. Writing $a_{j}:=e_{j} \Psi$ for $j=1, \ldots, n$, we have the zonotope

$$
Z=Z\left(\lambda_{1}, \ldots, \lambda_{n}\right):=C \Psi=\sum_{i=1}^{n} \frac{1}{2} \lambda_{i}\left[-a_{i}, a_{i}\right]
$$

in $\mathbb{Y}$. It is an elementary exercise in linear algebra to show that (up to translation) an arbitrary zonotope $Z \subseteq \mathbb{Y}$ admits a representation of this form (for a suitable choice of orthonormal basis $\left(e_{1}, \ldots, e_{n}\right)$ and scalars $\left.\lambda_{1}, \ldots, \lambda_{n}\right)$.

The basis dual to $\left(\lambda_{1} e_{1}, \ldots, \lambda_{n} e_{n}\right)$ is $\left(\lambda_{1}^{-1} e_{1}, \ldots, \lambda_{n}^{-1} e_{n}\right)$, from which we obtain another box

$$
\bar{C}=C\left(\lambda_{1}^{-1}, \ldots, \lambda_{n}^{-1}\right):=\sum_{i=1}^{n} \frac{1}{2} \lambda_{i}^{-1}\left[-e_{i}, e_{i}\right] .
$$

Thus, if we define $\bar{a}_{j}:=e_{j} \Phi^{*}$ for $j=1, \ldots, n$, then, by definition, the zonotope associated with $Z$ is

$$
\bar{Z}=\bar{Z}\left(\lambda_{1}^{-1}, \ldots, \lambda_{n}^{-1}\right):=\bar{C} \Phi^{*}=\sum_{i=1}^{n} \frac{1}{2} \lambda_{i}^{-1}\left[-\bar{a}_{i}, \bar{a}_{i}\right]
$$

in $\mathbb{X}$. (For more details about associated zonotopes, see [9] and [20]; note that $\left(\bar{a}_{1}, \ldots, \bar{a}_{n}\right.$ ) is a linear transform of $\left(a_{1}, \ldots, a_{n}\right)$.)

Now consider a typical edge $E$ of $\operatorname{fib}(C ; \Phi)$. A contribution to $E$ (there may be more than one) arises from an $(m+1)$-face of $C$, say, without loss of generality, one of the translates of $J:=\sum_{i=1}^{m+1} \frac{1}{2} \lambda_{i}\left[-e_{i}, e_{i}\right]$. Let $\widehat{J}:=\sum_{i=m+2}^{n} \frac{1}{2} \lambda_{i}^{-1}\left[-e_{i}, e_{i}\right]$ be the orthogonal box giving the complementary faces of $\bar{C}$. We have seen that the volume ratio $\kappa(J, \Psi)$ of the projection of $J$ under $\Psi$ is the same as that of $\widehat{J}$ under $\Phi^{*}$, namely, $\kappa\left(\widehat{J}, \Phi^{*}\right)$. Moreover, $\widehat{J} \Phi^{*}$ spans the subspace orthogonal to $E$, and the union of the normal cones to the family of edges of fib $(C ; \Phi)$ parallel to $E$ is just this subspace. This tells us two things. First, as we have said, $\operatorname{fib}(C ; \Phi)$ is itself a zonotope. Second, this zonotope is the sum of line segments, the typical one of which is orthogonal to $\widehat{J} \Phi^{*}$, and of length

$$
\kappa(J, \Psi) \operatorname{vol}_{m+1}(J)=\lambda_{1} \cdots \lambda_{m+1} \kappa(J, \Psi) .
$$


On the other hand, two translates of $\widehat{J} \Phi^{*}$ are components of opposite facets of the associated zonotope $\bar{Z}$, and therefore contribute an orthogonal segment of length

$$
\operatorname{vol}_{n-m-1}\left(\widehat{J} \Phi^{*}\right)=\lambda_{m+2}^{-1} \cdots \lambda_{n}^{-1} \kappa\left(\widehat{J}, \Phi^{*}\right)
$$

to the projection body $\Pi(\bar{Z})$ of $\bar{Z}$.

Comparing these two expressions, and using $\kappa\left(\widehat{J}, \Phi^{*}\right)=\kappa(J, \Psi)$, we come to the following conclusion:

Proposition 9.1. With the notation of this section,

$$
\operatorname{fib}(C ; \Phi)=\lambda_{1} \cdots \lambda_{n} \Pi(\bar{Z}) .
$$

Of course, this exhibits $\operatorname{fib}(C ; \Phi)$ as a homogeneous polynomial of degree $m+1$ in $\lambda_{1}, \ldots, \lambda_{n}$. Note that we can now proceed to the limit, and allow $\lambda_{j}=0$ for any $j$.

\section{References}

1. L.J. Billera, I.M. Gel'fand and B. Sturmfels, Duality and minors of secondary polyhedra. J. Combin. Theory Ser. B 57 (1993), 258-268.

2. L.J. Billera and B. Sturmfels, Fiber polytopes. Ann. of Math. 135 (1992), 527-549.

3. L.J. Billera and B. Sturmfels, Iterated fiber polytopes. Mathematika 41 (1994), 348-363.

4. I.M. Gel'fand, A.V. Zelevinskiı̌ and M.M. Kapranov, Newton polytopes of principal A-determinants. Soviet Math. Dokl. 40 (1990), 287-281.

5. G. Green, An Essay on the Application of Mathematical Analysis to the Theories of Electricity and Magnetism. Privately published (Nottingham, 1828). (See also J. Reine Angew. Math. 39 (1850), 73-89; 44 (1852), 356-374; 47 (1854), 161-221.)

6. B. Grünbaum, Convex Polytopes. Wiley-Interscience, London, 1967. (Second edition prepared by V. Kaibel, V.L. Klee and G.M. Ziegler, Graduate Texts in Mathematics, Springer, New York, 2003.)

7. P.M. Gruber and J.M. Wills (editors), Handbook of Convex Geometry. North-Holland, Amsterdam, 1993.

8. J. McDonald, Fractional power series solutions for systems of equations. Discrete Comput. Geom. 27 (2002), 501-529.

9. P. McMullen, On zonotopes. Trans. Amer. Math. Soc. 159 (1971), 91-109.

10. P. McMullen, Weakly continuous valuations on convex polytopes. Arch. Math. 41 (1983), 555-564.

11. P. McMullen, Volumes of projections of unit cubes. Bull. London Math. Soc. 16 (1984), 278-280.

12. P. McMullen, Valuations and dissections. Chapter 3.6 in Handbook of Convex Geometry (eds. P.M. Gruber and J.M. Wills), pp. 933-988. Amsterdam, North-Holland, 1993.

13. P. McMullen, Weights on polytopes. Discrete Comput. Geom. 15 (1996), 363-388.

14. P. McMullen, Polytope algebras, tensor weights and piecewise polynomials. In Intuitive Geometry (ed. L. Szabo), pp. 159-177. Bolyai Society Mathematical Studies, Vol. 6. János Bolyai Mathematical Society, Budapest, 1997.

15. P. McMullen, Fibre tilings. Mathematika 50 (2003), 1-33.

16. P. McMullen, Valuations and tensor weights on polytopes (in preparation).

17. T. Michiels and R. Cools, Decomposing the secondary Cayley polytope. Discrete Comput. Geom. 23 (2000), 367-380.

18. A.V. Pukhlikov and A.G. Khovanskiĭ, Finitely additive measures of virtual polytopes. St. Petersburg Math. J. 4 (1993), 337-356 (trans. from Russian).

19. R. Schneider, Mixed polytopes. Discrete Comput. Geom. 29 (2003), 575-593.

20. G.C. Shephard, Combinatorial properties of associated zonotopes. Canad. J. Math. 24 (1974), 302-321.

21. G.M. Ziegler, Lectures on Polytopes. Springer, New York, 1995.

Received July 1, 2003, and in revised form March 12, 2004. Online publication September 23, 2004. 